\title{
Predicción del autoconcepto a partir de las emociones maternas en niños de edad escolar y diferencias según sexo
}

\author{
Santiago Resett ${ }^{1}$, Lisie Meier², Olinca García Sánchez ${ }^{3}$ y Olivia Katz ${ }^{4}$
}

\begin{abstract}
Artículo
Material original autorizado para la publicación en la revista Psicodebate. Facultad de Ciencias Sociales. Universidad de Palermo.

Recibido 04-11-2015 | Aceptado 12-02-2016
\end{abstract}

\section{Resumen}

El autoconcepto es un importante constructo dentro del sí mismo. Este cumple funciones protectoras, organizacionales y motivacionales. La presente investigación tuvo como objetivo general evaluar si las emociones positivas experimentadas por las madres predecían el autoconcepto de sus hijos con edades de entre 10 a 12 años. Asimismo, se buscó evaluar el autoconcepto de los niños y determinar si variaba según el sexo de ellos, por un lado, y si el sexo de los niños moderaba la relación entre las emociones maternas y el autoconcepto, por el otro. Fueron evaluados 160 sujetos: 80 madres y sus 80 respectivos hijos e hijas. Para la recolección de datos se utilizaron el Perfil de Autopercepción para Niños de Harter, el Cuestionario de Emociones Positivas para Adultos de Regner aplicado a las madres y un cuestionario sociodemográfico. A partir de una serie de regresiones lineales múltiples, se pudo comprobar que la experiencia de algunas emociones positivas maternales es un predictor significativo del autoconcepto que los niños tenían de sí mismos. También se pudo observar que el autoconcepto de los niños variaba según el sexo $F(4)=6.79, p<.01$, eta parcial al cuadrado $=8 \%$, pero sólo debido a que las mujeres se evaluaban más positivamente en competencia escolar y buen comportamiento. El sexo de los niños moderaba la relación entre entusiasmo/interés y los dominios escolar, físico y buen comportamiento, ya

1 Universidad Católica Argentina-CONICET - Argentina; santiago_resett@hotmail.com

2 Universidad Nacional de Formosa-CONICET - Argentina

3 Universidad Autónoma de Nuevo León - México

4 Universidad Católica Argentina - Argentina 
que dicha asociación era más significativa para las niñas. En las conclusiones se analizan los hallazgos y se brindan sugerencias para futuros estudios.

Palabras Clave: autoconcepto, emociones, madres, niños.

\title{
Prediction of self-concept in children by maternal emotion and sex differences
}

\begin{abstract}
Self-concept is an important aspect of self. It relates to mental health, selfregulation, and motivation. The present research aims to evaluate whether the experience positive emotions of mothers predicted self-concept in children aged 10 to 12 years. Another purpose was determinate whether self-concept in children varied by their gender, on one hand, and whether gender of child was a moderator in this respect, on the other. The advantage of this study was evaluated both mothers and their children. A descriptive-correlational, cross-sectional and field study was conducted; 80 children and 80 mothers where assessed. Participants completed Harter Self-Perception Profile for Children, Regner Positive Emotions Questionnaire for Adults Regner, and socio-demographic questions, respectively. After performing multiple regression analyses, results suggested that the experience of some positive maternal emotions was related to positive concept in children. It was also found that gender introduced a small differences in selfconcept of children $F=6.79, p<.01$, eta parcial al cuadrado $=8 \%$. Females only scored better than males in scholastic competence and behavioral conduct. The gender of the child moderated the association among interest/enthusiasm and scholastic, physical, and behavioral domains due to more significant associations for female in this respect. In the conclusion findings are discussed, limitations of the study are pointed out, and suggestions for future studies are provided.
\end{abstract}

Keywords: self-concept, emotions, mothers, children. 
Desde la revolución cognitiva, las investigaciones sobre el desarrollo del sí mismo han florecido en distintas disciplinas A partir de dicha revolución, diferentes modelos y marcos teóricos han enfatizado uno u otro aspecto de este constructo. En el caso del sí mismo de niños y adolescentes, existen tres líneas diferentes de investigación. La primera se concentra en la autoestima (cuán positiva o negativamente se percibe a sí mismo un sujeto). La segunda, en los cambios del autoconcepto, esto es, en las ideas que el niño o adolescente tiene de sus rasgos y atributos. La tercera, en el desarrollo de la identidad -el sentido de quién es uno, de dónde viene y hacia dónde va- (Facio, Resett, Mistrorigo \& Micocci, 2006). En lo referente al desarrollo de los niños y adolescentes, las estructuras del sí mismo sirven para alcanzar metas y para proveer criterios y guías que ayudan a la autorregulación (Harter, 1999, 2006, 2012; Higgins, 1991, 1996). Del mismo modo, el sí mismo es un aspecto vital de la salud psicológica de un individuo, ya que se sabe que un sentimiento de valía personal elevado es un componente esencial de la salud mental (Harter, 2012). Uno de los conceptos más relevantes con relación al sí mismo es el autoconcepto, el cual refiere a un juicio o evaluación sobre cuán positiva o negativamente se percibe un sujeto en un dominio de su vida (Harter, 1999, 2006). El autoconcepto tiene la función de organizar las expectativas y brindar una guía para facilitar la interpretación de las experiencias personales. Asimismo, tiene una gran relevancia para la autorregulación y la motivación (Harter, 1999, 2006); también cumple una función para la autorregulación de la conducta en las actividades sociales y cognitivas (Casullo, 1990). Finalmente, ejerce un papel protector para la salud mental, ya que si uno se juzga positivamente en distintos aspectos de su vida, esto puede asociarse con un mayor bienestar psicológico (Harter, 2006).

El autoconcepto se distingue claramente de la autoestima, que es un juicio global sobre cuán valioso o positivo se percibe uno. Las investigaciones de Harter (1999, 2006, 2012) a lo largo de la década del ochenta comprobaron que los niños discriminan -además de la autoestima- cinco áreas de su vida (dominio comportamental, escolar, social, físico y deportivo) e informan niveles de adecuación diferentes, según el dominio de que se trate. Así, los niños de edad escolar -principalmente los niños mayores (entre 8 y 11 años)- pueden autodescribirse en distintos dominios de sus vidas ("inteligente", "bueno en los deportes", entre otros); tienen mayor capacidad de coordinar dichas representaciones ("bueno en la escuela, pero no tan bueno en los deportes") y pueden tener en cuenta no sólo sus evaluaciones, sino también las de los demás, como padres y pares (Harter, 1999, 2006).

El sexo (masculino o femenino) es una de las distinciones sociales más tempranas que hacen los niños: hay conductas o comportamientos más propios de varones o mujeres (Baron, Schmader, Cvencek \& Meltzoff, 2014). Se sabe 
que el sexo de los sujetos - debido a factores sociales y/o biológicos (Leaper \& Friedman, 2007)- es una variable que introduce diferencias en diversos aspectos del desarrollo psicosocial, como lo es el caso de la autopercepción (Kling, Hyde, Showers \& Buswell, 1999). En lo referente al autoconcepto, Hattie (1992) encontró que -aunque en la mayoría de las muestras australianas el sexo no era un factor tan importante- se evidenciaban algunas diferencias pequeñas. Feingold y Mazzella (1998) hallaron en todas las edades una moderada disparidad que se evidenciaba en la mayor puntuación de los varones en la dimensión apariencia física. Asimismo, Harter $(1985,1988,2006)$ informó diferencias según sexo en favor de los varones, en el caso de la apariencia física y la competencia deportiva, tanto en la niñez como en la adolescencia. En Noruega, Wichstrom (1998) encontró que los varones puntuaban más alto en apariencia física, competencia atlética, atractivo amoroso y competencia escolar. En nuestro país, algunos estudios en la niñez (Rodríguez, Moreno \& Resett, 2012) hallaron diferencias en competencia escolar y buen comportamiento a favor de las mujeres, mientras que los varones puntuaron más alto en competencia deportiva y social. También Molina, Raimundi, López, Cataldi y Bugallo (2011) hallaron diferencias de sexo en niños de edad escolar, ya que los niños puntuaban más alto en competencia deportiva, mientras que las niñas lo hacían en buen comportamiento. Del mismo modo, Molina y Raimundi (2011) detectaron que la apariencia física era el mejor predictor de la autoestima global para los niños, mientras que, en el caso de las niñas, lo era la aceptación social.

Así, una vasta literatura indica que los niños de edad escolar distinguen distintos dominios del autoconcepto y que la diferencia más grande entre varones y mujeres en la niñez se da en la dimensión competencia deportiva (Jacobs, Lanza, Osgood, Eccles \& Wigfield, 2002). Por lo tanto, la presente investigación evaluó si niños y niñas diferían en distintos dominios del autoconcepto.

Una importante área de estudio reciente, tanto para la psicología clínica como la psicopatología del desarrollo, son las emociones. Fredrickson $(1998,2001)$ sostiene que las emociones son tendencias de respuesta de varios componentes, que se despliegan durante períodos de tiempo relativamente cortos ante un estímulo, y que generan cambios conductuales, fisiológicos y cognitivos. Las emociones se diferencian del afecto de varias maneras: a) en primer lugar, las emociones surgen típicamente alrededor de alguna circunstancia o estímulo, mientras que el afecto es a menudo flotante o sin objeto; b) en segundo lugar, las emociones son usualmente breves e implican a los sistemas de componentes múltiples descritos anteriormente, mientras que el afecto es de más larga duración y puede manifestarse sólo a nivel de la experiencia subjetiva; c) y, por último, podrían agruparse dentro de diferentes categorías de acuerdo con la atracción-rechazo o a su valencia positiva-negativa, por ejemplo (Lucas, Diener \& Larsen, 2003). 
Si bien no hay un consenso en la clasificación de las emociones, y estas son todavía objeto de un profundo debate (Nesse \& Ellsworth, 2009), una clasificación típica de las emociones consiste en separarlas en primarias y secundarias (o sociales); las primeras incluyen miedo, ira, tristeza, asco, alegría, sorpresa y -posiblemente- desprecio (Ekman, 1972). Son universales, con una base genética, con una expresión facial distintiva, sirven para la supervivencia y serían compartidas por los humanos con algunos animales superiores, como los homínidos (chimpancés, gorilas, entre otros). Las secundarias, como orgullo, culpa, gratitud, vergüenza, entre otras, serían propiamente humanas; implican la conciencia de uno mismo, y lentamente comenzarían a emerger a partir de los 2-3 años de vida (Harter, 1999).

Por otro lado, pero siguiendo la línea de las emociones positivas, Seligman (2003, como se citó en Carr, 2007) establece que ellas se clasifican en tres categorías: las relacionadas con el pasado, las relacionadas con el presente y las relacionadas con el futuro. Las relacionadas con el futuro incluyen el optimismo, la esperanza, la seguridad, la fe y la confianza. Las principales emociones positivas asociadas con el pasado son la satisfacción, la realización personal, el orgullo y la serenidad. En cuanto a las relacionadas con el presente, hay dos clases diferentes: los placeres (que pueden ser corporales y superiores), que son momentáneos, y las gratificaciones, que son más duraderas.

Se sabe que la familia es un socializador primario por excelencia (Bowlby, 1982; Collins, Maccoby, Steinberg, Hetherington, \& Bornstein, 2000; Davidov \& Grusec, 2006). En lo referente a la relación entre la calidad del ambiente familiar y las autoevaluaciones, la literatura es sólida. Glasner (1960, como se citó en Casullo, 1990) plantea que del sistema familiar y de su íntima interacción con el niño, surgen los llamados "autoconceptos primarios", los cuales sirven como sustento y base para el desarrollo de los "autoconceptos secundarios", los cuales continuarán adquiriéndose con relación a la interacción que se pueda ir dando extrafamiliarmente. Es de esta forma que, en gran medida, la adquisición adaptada y correcta del autoconcepto supone o conlleva la aprobación y apoyo de parte de los adultos significativos (Casullo, 1990). Esto se percibe más claramente en los niños mayores (8 a 11 años), quienes ya pueden -en cierta medida- integrar sus autoevaluaciones con las brindadas por los demás, como padres, docentes y pares (Harter, 1999, 2012). De este modo, está bien documentado que los padres y madres que son cálidos, responsables y que brindan apoyo instrumental y emocional tienen hijos con una autoevaluación positiva en diferentes dominios (Harter, 2006, 2012); ya Bowlby (1982) y Erikson (1983) postulaban que los padres emocionalmente cálidos y sensibles facilitaban en los hijos el desarrollo de un sí mismo valioso y competente. Pocas investigaciones -incluso en los países del primer mundo- han investigado si la expresión de distintas 
emociones maternas se asocia con un autoconcepto más positivo en sus hijos, ya que la mayoría de los estudios se centraron en la calidez y disponibilidad emocional de los cuidadores primarios (e.g., Harter, 2006). Pocos trabajos evaluaron si el simple hecho de expresar un mayor nivel de emociones positivas en los progenitores (por ejemplo, madres) se asociaba con un mayor autoconcepto de los hijos. Se sabe que madres y padres se comportan de modo diferente con sus hijos e hijas (Leaper \& Friedman, 2007; Parke \& Gauvain, 2009), al seguir los estereotipos de sexo (por ejemplo, juguetes o actividades más "masculinos" o "femeninos" que escogen, según el sexo de su hijo), al ser más protectores con sus hijas que hijos, o al fomentar una menor independencia en las niñas. También dichas diferencias según el sexo se perciben en que ambos progenitores facilitan una mayor expresión y calidez emocional en sus hijas: "boys do not cry". Consecuentemente, el presente trabajo pretende hacer una contribución a la literatura científica al examinar si algunas emociones positivas maternas se asocian con el autoconcepto de sus hijos en distintos dominios, a partir del informe de las madres (emociones positivas) y de sus hijos (autoconcepto), lo cual evita que se aumenten las correlaciones artificialmente por la varianza del método de recolección de datos compartido -mostrando mayores asociaciones que las empíricamente existentes-. Además, busca determinar si las emociones positivas maternas influían de modo diferente en el autoconcepto, según el sexo de los niños, esto es, si el sexo de los hijos modera dicha relación.

Teniendo en cuenta todo lo anteriormente planteado, la presente investigación trató de responder las siguientes preguntas: ¿varía el autoconcepto según el sexo de los niños?, ¿algunas emociones positivas (alegría, interés/entusiasmo, tranquilidad, gratitud y compasión) experimentadas por las madres predicen el autoconcepto del niño de 10-12 años de edad? y ¿modera el sexo de los niños la relación entre la expresión de emociones positivas maternas y el autoconcepto?

\section{Objetivos}

\section{Objetivo general}

Conocer la relación de la experiencia de emociones positivas maternas con el autoconcepto del niño de entre 10-12 años y establecer si existen diferencias en el autoconcepto según el sexo de los niños

\section{Objetivos específicos}

Explorar el autoconcepto en los niños de entre 10-12 años.

Identificar si existen diferencias en el autoconcepto de los niños según el sexo.

Determinar si la experiencia de emociones positivas maternas predice el autoconcepto de los niños y si el sexo de los niños modera dicha predicción. 


\section{Método}

\section{Participantes}

La selección de sujetos se efectuó mediante un muestreo no probabilístico intencional, en uno de sus ámbitos más cotidianos: la escuela. Fueron incluidos 80 alumnos de escuelas públicas y privadas del nivel primario de la ciudad de Paraná, Entre Ríos. Además, se incluyó como participantes a las madres de cada uno de los alumnos seleccionados como parte de la muestra.

La muestra de las madres estuvo compuesta por 80 sujetos, quienes tenían entre 25 y 55 años de edad $(M=38.63 ; D E=7.02)$. La muestra de los niños de entre 10 y 12 años de edad $(M=10.75 ; D E=.78)$ estuvo compuesta por 80 sujetos, 40 de sexo femenino y 40 de sexo masculino, los cuales asistían a los niveles de educación primaria entre cuarto y sexto grado.

\section{Materiales}

Los instrumentos seleccionados para la obtención de los datos, fueron:

Perfil de Autopercepción para Niños (Harter, 1985). Creado por Harter (1985) y adaptado a la Argentina por Molina y otros (2011). La autora de dicho cuestionario diseñó este instrumento para evaluar la autoestima global junto con las percepciones de competencia o adecuación a nivel cognitivo, social y físico. Dichos dominios del autoconcepto son: Competencia escolar o académica: explora la percepción del niño de sus competencias o habilidades en el contexto del rendimiento académico. Los ítems que la evalúan son el 5, 11, 17, 23, 29 y 35. Aceptación social: mide el grado en que el sujeto se percibe aceptado por su grupo de pares o se siente popular. Los ítems que lo miden son el 4, 10, 16, 22, 28 y 34. Competencia deportiva: mide la capacidad para los deportes o actividades al aire libre. Sus ítems son 1, 7, 13, 19, 25 y 31. Apariencia física: explora el grado en que el niño se encuentra conforme con la forma en que luce, le agrada su altura, cara y peso. Los ítems que lo evalúan son el 3, 9, 15, 21, 27 y 33. Buen Comportamiento/Conducta: evalúa el grado en que al niño le agrada cómo se comporta, realiza lo correcto y actúa de la forma en que los demás esperan que se comporte. Sus ítems son 2, 8, 14, 20, 26 y 32. Molina et al. (2011) hallaron que en la muestra argentina dichos dominios explicaban el 51.30 $\%$ de la varianza total. También Molina et al. (2011) detectaron en sus estudios as adecuadas: .84, .79, .81, .79 y .70, respectivamente. En el presente trabajo, los índices de consistencia interna fueron $\alpha$ s.84, $.86, .80, .72$ y .75, respectivamente. El cuestionario está destinado a niños de 8 a 12 años y se compone de 36 ítems con cuatro opciones de respuesta, las cuales consisten en dos frases contrapuestas que muestran dos grupos de niños con autopercepciones opuestas, las cuales fueron diseñadas así para disminuir la deseabilidad social. Los niños deben elegir en 
primer lugar a qué grupo se parecen y, en segundo lugar, en qué grado se parecen a ese grupo, total o parcialmente (Molina et al., 2011). El estilo de respuesta es de tipo Likert, con cuatro opciones por cada ítem del perfil, con puntajes que varían de 1 a 4 ("yo no soy realmente asi" hasta "yo soy realmente asi"). Harter (1985) diseñó un formato original de respuesta que solicita optar primero entre la afirmación de la derecha y la de la izquierda (por ejemplo, ella/él ¿se considera que es bueno para los deportes?) y luego decidir cuál nivel de intensidad refleja mejor su percepción (¿se siente realmente así o sólo algo así?). Intentaba así legitimar ambas opciones y mejorar un importante problema de anteriores escalas: la tendencia a dar respuestas socialmente deseables. A mayor puntaje, mayor será el nivel del constructo medido, con puntajes que van de 1 a 4 (Atienza, Balaguer \& Moreno 2002).

Cuestionario de Emociones Positivas (Regner, 2009). Para evaluar la experiencia de emociones positivas en los adultos se utilizó el instrumento creado por Regner (2009), el cual consta de 35 ítems distribuidos en cinco factores que evalúan las siguientes emociones positivas: Alegría, Interés/ Entusiasmo, Tranquilidad, Gratitud y Compasión. En cuanto a la validez factorial del constructo, la autora (Regner, 2009) confirmó la presencia de los cinco factores propuestos. Dichos factores explicaban un $47.94 \%$ de la variancia total. A continuación, se procede a conceptualizar cada dimensión: Factor Alegría: ítems 5, 8, 12, 18, 27, 30 y 33. Es una intensa emoción positiva que se desencadena por hechos satisfactorios de la vida. Hace referencia a un estado de contentamiento general, de regocijo y diversión, cuya expresión máxima es la risa (Lazarus, 2000). Factor Tranquilidad: ítems 4, 6, 10, 13, 20, 25 y 32. Es una reacción profunda de expresión o sensación de confianza y paz interior. Puede trascender más allá a eventos externos, incluso experimentarse en circunstancias de adversidad (Roberts \& Cunningham, 1990). Factor Compasión: ítems 1, 11, 16, 21, 23, 31 y 34. Es la compresión o entendimiento del estado emocional afectivo ajeno, relacionada con un deseo de reducir o aliviar el sufrimiento o malestar del otro (Lazarus \& Lazarus, 2000). McKay y Fanning (1991) establecen que está compuesta por tres dimensiones básicas: la comprensión, la aceptación y el perdón, que pueden estar dirigidas hacia uno mismo o hacia los demás. Factor Gratitud: ítems 3, 7, 9, 15, 26, 29 y 35. Es un constructo de gran importancia social (Lazarus, 2000). Se experimenta esta emoción cuando: (a) se obtiene un beneficio que es percibido como positivo, (b) dicho beneficio no se obtuvo por esfuerzo propio, sino que (c) ha sido entregado voluntariamente por un benefactor (Emmons \& McCullough, 2003). Factor Interés-Entusiasmo: ítems 2, 14, 17, 19, 22, 24 y 28. Es aquella emoción que se experimenta más regularmente, relacionada con la atención, percepción y motivación (Fredrickson, 2000). Se encuentra asociada con el deseo de atender a un evento o situación que es valorada como importante 
(Ellworth \& Smith, 1988, como se citó en Regner, 2009). La confiabilidad total del instrumento resultó satisfactoria, según Regner (2009), $\alpha=.89$, al igual que en cada uno de sus factores. La autora detectó para el factor Alegría un $\alpha=.90$; para el factor Interés-Entusiasmo obtuvo $\alpha=.81$; para el factor Tranquilidad, $\alpha=.84$; para el factor Gratitud, $\alpha=.81$ y para Compasión, $\alpha=.84$. En el presente trabajo las $\alpha$ s fueron: $.87, .87, .82, .92$ y .89, respectivamente. El instrumento posee una modalidad de respuesta de tipo Likert, en donde las alternativas a responder son: "fuertemente desacuerdo" (1), "desacuerdo" (2), "neutral" (3), "de acuerdo" (4) y "fuertemente de acuerdo" (5); las cuales indicarían que, a mayor puntuación positiva (fuertemente de acuerdo), mayor será el nivel del constructo medido.

\section{Procedimiento para la recolección de los datos}

Para la recolección de datos se solicitó a los directivos de las instituciones el acceso a los colegios. Se les envió a las madres una nota en la cual se les explicó los motivos de la investigación y se les entregó el consentimiento informado para evaluar a sus hijos y, a su vez, el consentimiento informado para su participación. Las madres que estuvieron dispuestas a colaborar fueron convocadas a una reunión en la cual se les aplicó el cuestionario. Una vez tomadas las pruebas a ellas, se procedió a suministrar los cuestionarios pertinentes a los niños en horas de clases; se aseguró anonimato, confidencialidad y participación voluntaria.

\section{Procedimiento para el análisis de los datos}

El análisis de datos se llevó a cabo con el programa Statistical Package for the Social Sciences (SPSS) 20. Se llevaron a cabo estadísticos descriptivos, como medias, desvíos típicos, entre otros, e inferenciales, como MANOVAS análisis de perfiles y regresiones lineales múltiples y jerárquicas.

\section{Resultados}

En relación con el primer objetivo, en el cual se pretendía evaluar el autoconcepto de los niños, se halló: en competencia escolar una media de 3.23; en aceptación social una media 3.21; en competencia deportiva una media de 3.21 ; en apariencia física una media de 3.26 y en buen comportamiento una media de 3.34. Los resultados pueden observarse en la tabla 1. 
Tabla 1.

Mínimos, máximos, medias y desvíos estándar de las dimensiones de autoconcepto en niños de 10-12 años

\begin{tabular}{lccccc}
\hline Autoconcepto & $\boldsymbol{n}$ & Mínimo & Máximo & M & DE \\
\hline Competencia escolar & 80 & 1.50 & 4.00 & 3.23 & .62 \\
Aceptación social & 80 & 1.33 & 4.00 & 3.21 & .64 \\
Competencia deportiva & 80 & 1.33 & 4.00 & 3.21 & .59 \\
Apariencia física & 80 & 1.50 & 4.00 & 3.26 & .74 \\
Comportamiento & 80 & 1.50 & 4.00 & 3.34 & .67 \\
\hline
\end{tabular}

Con respecto al segundo objetivo planteado, el de identificar si existían diferencias en el autoconcepto según el sexo de los niños, al analizar los resultados se pudo observar la distribución de las medias obtenidas para las dimensiones del autoconcepto en función del sexo, como se ve en la tabla 2.

Tabla 2.

Distribución de medias y desvios típicos del autoconcepto según el sexo de los niños de $10-12$ años

\begin{tabular}{|c|c|c|c|c|c|}
\hline & Sexo & $M$ & $D E$ & $F$ & $p$ \\
\hline \multicolumn{6}{|l|}{ Competencia escolar } \\
\hline & Varón & 3.04 & .77 & 8.04 & .006 \\
\hline & Mujer & 3.42 & .33 & & \\
\hline & Total & 3.23 & .62 & & \\
\hline \multicolumn{6}{|l|}{ Aceptación social } \\
\hline & Varón & 3.17 & .70 & .39 & .531 \\
\hline & Mujer & 3.26 & .59 & & \\
\hline & Total & 3.21 & .64 & & \\
\hline \multicolumn{6}{|l|}{ Competencia deportiva } \\
\hline & Varón & 3.12 & .64 & 1.76 & .187 \\
\hline & Mujer & 3.30 & .52 & & \\
\hline & Total & 3.21 & .59 & & \\
\hline \multicolumn{6}{|l|}{ Apariencia física } \\
\hline & Varón & 3.12 & .81 & 3.15 & .080 \\
\hline & Mujer & 3.41 & .64 & & \\
\hline & Total & 3.26 & .74 & & \\
\hline \multicolumn{6}{|l|}{ Comportamiento } \\
\hline & Varón & 3.12 & .79 & 9.64 & .003 \\
\hline & Mujer & 3.57 & .43 & & \\
\hline & Total & 3.34 & .67 & & \\
\hline
\end{tabular}


Para observar si el sexo introducía diferencias, se realizó un MANOVA análisis de perfiles con las escalas de autoconcepto como variables dependientes y el sexo de los niños como factor entre sujetos. Así, se observó que no se hallaron diferencias en la forma, pero sí en la altura de los perfiles $\mathrm{F}(4)=6,79, \mathrm{p}<.01$, eta parcial al cuadrado $=8 \%$; con un intervalo de confianza al $95 \%$ se vio que las niñas puntuaban más positivamente en competencia escolar y buen comportamiento en comparación con los niños, como se muestra en la tabla 2.

En la tabla 3 se muestran los puntajes en las emociones positivas maternas. Se observaron los siguientes resultados: en alegría una media de 3.76; en tranquilidad una media de 3.75; en compasión una media de 3.96; en gratitud una media de 4.27 y en entusiasmo una media de 3.93 , como se ve en la tabla 3.

\section{Tabla 3.}

Mínimos, máximos, medias y desvíos estándar en las emociones positivas de las madres de niños de 10-12 años.

\begin{tabular}{lccccc}
\hline Emociones positivas & $n$ & Mínimo & Máximo & $\boldsymbol{M}$ & $\boldsymbol{D E}$ \\
Alegría & 80 & 2.29 & 5.00 & 3.76 & .69 \\
Tranquilidad & 80 & 1.86 & 5.00 & 3.75 & .75 \\
Compasión & 80 & 1.00 & 5.00 & 3.96 & .69 \\
Gratitud & 80 & 1.00 & 5.00 & 4.27 & .73 \\
Entusiasmo & 80 & 1.00 & 5.00 & 3.93 & .74 \\
\hline
\end{tabular}

Con respecto al tercer objetivo, el de analizar si las emociones positivas de las madres predecían el autoconcepto de sus hijos, se llevaron a cabo regresiones lineales múltiples en la cuales se colocaron las emociones maternas como predictores y cada uno de los dominios del autoconcepto como variable dependiente. En la tabla 4 se presentan los resultados de las ecuaciones de predicción. 


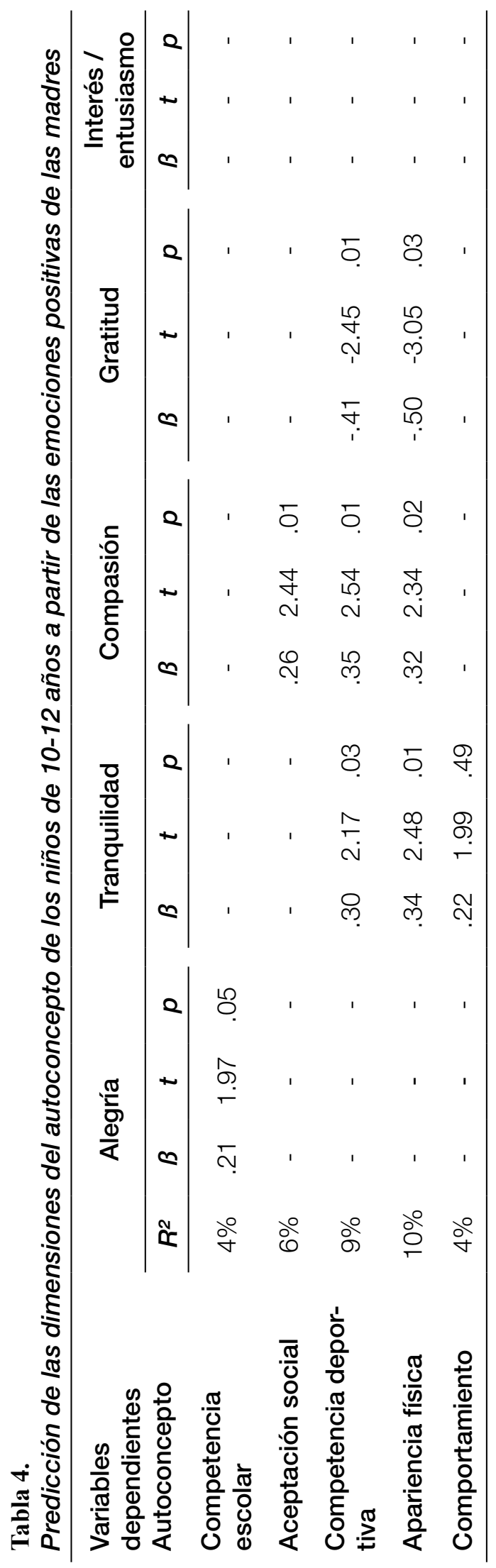


Como se percibe en la tabla 4, se pudo observar que la competencia escolar fue predicha por la dimensión alegría, ya que explicaba un 4\% de la varianza. En cuanto a la dimensión del autoconcepto denominada aceptación social, se observó que la compasión predijo un $6 \%$ de la varianza. En lo referente a la competencia deportiva, la varianza de la misma fue predicha en $9 \%$ por tranquilidad, compasión y gratitud -en sentido negativo-. Para la dimensión apariencia física, nuevamente tranquilidad, compasión y gratitud -en sentido negativo- predijeron un $10 \%$ de la varianza. Finalmente, para buen comportamiento, se observó que fue predicha en un 4\% por el factor tranquilidad -aunque no alcanzaba la significación, era el mejor predictor-.

Finalmente, para determinar si el sexo de los niños era un factor que moderaba la predicción del autoconcepto, se llevaron a cabo regresiones jerárquicas, por lo que se colocaron en el primer bloque las emociones maternas, en el segundo bloque el producto del puntaje de cada emoción materna por el sexo (varón $=0$, mujer $=1$ ) y el puntaje de autoconcepto como variable dependiente. Los resultados indicaron que existía interacción para competencia escolar, ya que $R^{2}$ se incrementaba significativamente de $3 \%$ a $16 \% \mathrm{p}<.009$ y la variable significativa del segundo bloque era el producto del entusiasmo por el sexo $\beta=2.53 t=2.81 p<.006$. Los resultados también mostraron que existía interacción para apariencia física, ya que $R^{2}$ se incrementaba significativamente de $9 \%$ a $19 \% p<.02$ y la variable significativa era el producto del entusiasmo por el sexo $\beta=2.54 t=2.87 p<.005$. Por último, se detectó que existía interacción para buen comportamiento, ya que $R^{2}$ se incrementaba significativamente de $2 \%$ a $18 \% \mathrm{p}<.004$, con la tranquilidad $\beta=2.24 t=-2.35 p<$ .02 , y con el entusiasmo $\beta=1.68 t=1.88 p<.05$ como predictores significativos. Esto es, en dichos dominios del autoconcepto, que un mayor entusiasmo se asociaba con una evaluación más positiva en el área escolar, física y buen comportamiento en el caso de las mujeres. En cambio, una mayor tranquilidad se relacionaba con juzgar un comportamiento más negativo en el caso de los varones.

\section{Discusión}

El objetivo general que guió a esta investigación fue conocer si la experiencia de las emociones positivas maternas predecía el autoconcepto de sus hijos.

Con relación al objetivo primero, explorar el autoconcepto de los niños (competencia escolar, aceptación social, competencia deportiva, apariencia física y buen comportamiento), se halló que los mismos poseían un autoconcepto ligeramente alto, el cual se evidenció en la mayoría de las respuestas elegidas, ya que las medias iban de 3.21 a 3.34. Esto puede deberse, tal vez, a que los niños a esta edad aún no tienen un sí mismo totalmente desarrollado, integrado y no pueden contrastarlo con los estereotipos y situaciones conflictivas más comunes 
de la adolescencia. Los niños de esta edad pueden juzgarse en diferentes dominios de su autoconcepto, integrar evaluaciones positivas y negativas y emplear la percepción del medio social para autopercibirse. Sin embargo, Harter (1999, 2012) señaló que generalmente los niños tienen un sesgo positivo y sobrestiman sus habilidades, debido -entre otras cuestiones- a sus limitaciones cognitivas (un pensamiento concreto) como a la deseabilidad social. En lo relativo al desarrollo cognitivo de los niños mayores (8 a 11 años), está sólidamente comprobado que, en comparación con los adolescentes, no poseen un pensamiento abstractohipotético, carecen de metacognición y su pensamiento está más centrado en el presente que en el futuro (Arnett, 2010), lo cual limita la evaluación del sí mismo (Harter, 2012). De este modo, sus evaluaciones son menos estables a través de las situaciones y el tiempo; menos realistas y su sí mismo menos integrado o coherente que el de un adolescente o adulto (Harter, 1999). Por ejemplo, a esta edad a un niño le cuesta - a diferencia de un adolescente- percibir que un evento o hecho puede tener connotaciones tanto positivas como negativas: "me gusta que mi madre me quiera, pero desearía que no sea tan entrometida con mis amigos".

Por otra parte, el autoconcepto ligeramente alto puede deberse a que la muestra era intencional y a que los niños evaluados pertenecían a sectores socioeconómicos medios. Tal vez en muestras de niños de sectores bajos y medio-bajos podría emerger un autoconcepto menos positivo debido a las carencias materiales, cognitivas, emocionales y sociales que experimentan. Se sabe que el nivel socioeconómico es un factor que afecta la percepción del sí mismo (Argyle, 1994).

Con respecto al objetivo segundo, el cual pretendió identificar si existían diferencias en el autoconcepto de los niños según el sexo, se comprobó que había diferencias en los niveles de autoconcepto, ya que en los dominios de competencia escolar y buen comportamiento las niñas se evaluaban más positivamente que los niños. En nuestro medio, también Rodríguez et al. (2012) hallaron dichas diferencias a favor de las niñas en estos aspectos, aunque los varones se evaluaban más positivamente en competencia deportiva y social. Del mismo modo, Molina et al. (2011) hallaron que las mujeres se evaluaban mejor en buen comportamiento, pero los varones puntuaban más alto en competencia deportiva. Que las diferencias hayan emergido sólo a favor de las niñas podría deberse a que, en la sociedad actual de la Argentina, la actitud machista ha decrecido y la brecha entre los sexos con respecto al trabajo, la educación, la participación política y la libertad sexual se ha ido estrechando. Cabe aclarar que esta muestra pertenecía a sectores socio-económicos medios. Es sabido que en los mismos las diferencias entre los sexos no son tan pronunciadas como en los sectores bajos o medios bajos, en los cuales la actitud machista es más fuerte (Argyle, 1994). Por ejemplo, en la presente muestra no se observaron diferencias a favor de los varones ni siquiera en competencia deportiva. 
Por otra parte, las mayores diferencias de sexo -y a favor de los varones- se detectan durante la adolescencia, principalmente debido a los cambios puberales y la hipótesis de la intensificación de los sexos: la diferenciación definitiva de qué es lo masculino y qué es lo femenino (Arnett, 2010; Steinberg, 2008). Como numerosas investigaciones mostraban, por ejemplo, en nuestro país las diferencias en autoconcepto entre mujeres y varones adolescentes eran mayores que las encontradas en los denominados países desarrollados, principalmente debido a que las mujeres adolescentes se evaluaban más negativamente que los varones en numerosos dominios, como hallaron Facio et al. (2006). Sin embargo, el presente diseño de investigación transversal no permite establecer si las diferencias en sólo dos áreas y a favor de las mujeres se deben a cuestiones evolutivas de la edad: ¿la diferencia es pequeña en la niñez y aumenta en la adolescencia a causa de los cambios puberales y la intensificación de sexo?; o, en cambio, este hecho se debe al efecto de la cohorte: ¿a medida que pasan los años la actitud machista va lentamente disminuyendo y los varones no se perciben más positivamente que las mujeres, como ocurría años atrás?

En lo referente a la experiencia de emociones positivas maternas -si bien no era un objetivo de la investigación-, se halló que la mayoría de los individuos optaban por la opción de respuesta de "estar de acuerdo" con las situaciones planteadas, ya que las medias iban de 3.75 a 4.27. Esto indicaba que la mayoría de las madres evaluadas se percibían como poseedoras de emociones mayormente positivas, las cuales se reflejaron en la obtención de resultados relativamente altos en las dimensiones de alegría, tranquilidad, compasión, interés o entusiasmo y gratitud. Dicha percepción, posiblemente, les otorgue tanto una experiencia satisfactoria de bienestar como recursos y herramientas adaptativas para enfrentar situaciones estresantes y adversas. Con relación a esto, Regner (2009) propuso que las emociones positivas constituyen un buen recurso psicológico para el afrontamiento de la amenaza, e influyen de forma positiva y directa sobre la confrontación de problemas y evaluación de situaciones. Sin embargo, dichas respuestas podrían también estar sesgadas por la deseabilidad social.

En cuanto al tercer objetivo, determinar si la experiencia de emociones positivas maternas predecía el autoconcepto de los niños, los resultados obtenidos permitían deducir que las emociones positivas relativamente altas de las madres podrían tener una correlación muy estrecha con la percepción de un autoconcepto relativamente elevado por parte de sus hijos, ya que se halló que el $4 \%$ de la varianza en competencia escolar era predicha por la alegría. En cuanto a la aceptación social, se observó que la compasión predecía un 6\%. En lo referente a la competencia deportiva, la varianza de la misma fue predicha en un $9 \%$ por la tranquilidad, compasión y gratitud (en sentido negativo). En la dimensión 
apariencia física, nuevamente tranquilidad, compasión y gratitud (en sentido negativo) predijeron un $10 \%$ de la varianza. Finalmente, buen comportamiento fue predicho en un $4 \%$ por tranquilidad. Como se ve, no sólo las experiencias de las emociones maternas eran predictores significativos, sino que tenían un tamaño entre mediano y grande, ya que iban de $4 \%$ a $10 \%$. Que gratitud se haya asociado negativamente con competencia deportiva y apariencia física, debe ser examinado en futuros estudios. Cabe aclarar que las dos variables se evaluaron con distintos informantes, por lo cual no están correlacionadas artificialmente por la varianza a raíz del método de recolección de datos compartido.

Está bien establecido que la relación con los cuidadores primarios juega un papel central para el desarrollo de un sí mismo seguro y positivo (Harter, 2006, 2012). Por ejemplo, se encontró que los cuidadores que son cálidos y emocionalmente sensibles tienen hijos con una autovaloración más segura y competente, esto es, una mejor autoestima y un autoconcepto más positivo; así, numerosos estudios en la Argentina, como los de Facio et al. (2006) y Richaud de Minzi (2006), detectaron que los hijos que percibían a sus padres más cálidos y comprensivos poseían una autovaloración más positiva.

Carr (2007) propone que las primeras experiencias de los niños con sus cuidadores, en especial sus madres, permiten que vayan adquiriendo con ellas una sensación de seguridad o seguridad muy marcada, las cuales condicionarán su autopercepción. Es así que cuando las madres responden con sensibilidad a sus necesidades, internamente se desarrolla una base segura y firme desde la cual se puede manifestar una gran confianza para explorar y vivir el mundo. Los niños que gozan de emociones positivas por parte de sus madres no sólo poseerán un vínculo más estrecho con ellas, sino que, además, desarrollarán mayor fortaleza y una mayor autoconfianza (Carr, 2007). En la presente investigación, esto se vio reflejado en el hecho de que los niños que tenían madres que expresaban una mayor alegría, tranquilidad y compasión, poseían una mejor autopercepción. Específicamente, que las madres expresen a sus hijos emociones positivas, tales como tranquilidad y compasión, hace que los niños tengan una mejor autoevaluación con respecto a su apariencia física. Del mismo modo, las mismas emociones, entiéndase por expresión de compasión y tranquilidad, se asocian en el niño con un autoconcepto más positivo en las competencias deportivas. Sin embargo, también se hallaron algunos efectos específicos de las emociones sobre los distintos dominios: por ejemplo, compasión se asociaba con aceptación social; alegría con competencia escolar y tranquilidad con buen comportamiento. Que interés/entusiasmo no haya sido un predictor significativo debe seguir siendo examinado; tal vez esto se deba a que dicho dominio impacta de modo diferente según el sexo del niño. Cuando se analizó si el sexo del hijo era un factor que moderaba la relación entre la expresión 
de las emociones positivas maternas y el autoconcepto de sus hijos, se halló que esto sucedía para competencia escolar, apariencia física y buen comportamiento. De este modo, se observó que para los tres dominios el entusiasmo/interés materno ejercía un impacto más fuerte para las hijas que los hijos. Tranquilidad estaba también moderada según el sexo, pero sólo para el buen comportamiento; sin embargo, en este caso una mayor tranquilidad expresada por las madres se asociaba con un peor comportamiento de los niños. Es sabido que padres y madres interactúan y socializan a sus hijos de modo diferente, según el sexo de ellos, principalmente siguiendo los estereotipos de qué es lo "femenino" y qué es lo "masculino". Es probable que las madres enfaticen más a sus hijas la importancia de andar bien en la escuela, de su aspecto físico o de cómo lucen (aspecto central de la feminidad en nuestra sociedad) y del comportarse bien (se sabe que un mal comportamiento es más tolerado en los niños). No obstante, se observó que una excesiva expresión de tranquilidad materna se asociaba con una peor conducta en sus hijos varones; tal vez debido a que ellos precisan un control más firme, aunque esto debe seguir siendo examinado en futuros estudios. Que el sexo de los hijos no introduzca diferencias en el autoconcepto social ni deportivo, puede deberse a que las madres esperan que tanto hijos e hijas sean agradables con los demás; a su vez, quizás para las madres el área deportiva no es un aspecto relevante para hijos ni hijas. Posiblemente el área deportiva es un dominio más de incumbencia paterna; el sexo del progenitor probablemente también sea un factor moderador, pero aquí sólo se evaluó a las madres. Que la moderación del sexo del hijo se haya materializado en entusiasmo/interés, debe ser evaluado en futuros trabajos. No obstante, esta emoción está muy vinculada con el grado de atención, interés, motivación y percepción, por lo cual se expresa muy regular y cotidianamente (Fredrikson, 2000). De este modo, no resultaba llamativo que en dicha emoción haya emergido el efector moderador, ya que es la que se experimenta más cotidianamente. Quizás las restantes emociones (alegría o compasión) se expresan en igual medida y ante las mismas situaciones para hijos e hijas. Por ejemplo, una madre puede mostrar mayor entusiasmo o dedicar mayor energía para que a su hija le vaya bien en la escuela, pero alegrarse del mismo modo y con la misma intensidad tanto si su hijo como hija traen buenas calificaciones.

Seligman (2007) propone que los niños que cuentan con un vínculo estrecho con sus madres disponen de más aptitudes para la resolución de problemas, como ser entusiasmo, curiosidad y perseverancia, lo que los lleva a percibir una mayor independencia y superación en casi todos los ámbitos que se han estudiado, experimentando una mayor emotividad positiva y un mejor autoconcepto. En lo relativo a las emociones positivas experimentadas por las madres, la vivencia de las mismas no sólo resulta gratificante por el sólo hecho de experimentarlas, sino que también estimulan una mayor y mejor relación con el mundo y su entorno 
(Seligman, 2007). En este caso particular, dicha expresión emocional positiva se vería reflejada en un autoconcepto más positivo en sus hijos e hijas, pero con efectos diferenciales según el sexo del hijo en algunos dominios.

\section{Limitaciones}

Las limitaciones del presente estudio fueron, en primer lugar, que los resultados estuvieron limitados por una muestra no intencional, pequeña y por las características sociodemográficas de la muestra. Debido a esto, los resultados no son generalizables a la población. También el tamaño no muy grande de la muestra no permite análisis más complejos como modelos estructurales con el programa AMOS.

Por otra parte, se trabajó sólo con niños escolarizados y de sectores socioeconómicos medios o altos. El panorama puede ser algo diferente en niños no escolarizados.

A su vez, los resultados también se restringieron a una investigación de tipo descriptivo-correlacional y transversal. Por tal motivo, no permite inferir la direccionalidad de la causalidad de las variables. Es posible también que los niños con un autoconcepto más positivo generen una mayor expresividad emocional positiva en sus madres.

Otra limitación es que se usó el autoinforme para evaluar el autoconcepto. En primer lugar, puede haber una distorsión en las respuestas debido a la deseabilidad social o sesgos en las respuestas por las limitaciones cognitivas de los niños o de comprensión lectora. Por otra parte, el formato de respuesta del Harter muchas veces dificulta el contestar, más aún en niños.

Finalmente, no se evaluaron las emociones paternas, sino solamente las maternas.

\section{Recomendaciones}

En primer lugar, se recomendaría ampliar la muestra de la investigación con un mayor número de sujetos, de tal modo que esta sea seleccionada aleatoriamente a fines de obtener resultados más generalizables.

En segundo lugar, se podría extender el estudio a la edad adolescente, para ver si las diferencias de sexo son más grandes en dicha etapa que en la niñez. Junto con esto, se propone el estudio de la investigación en conjunto con nuevas variables que puedan aportar información relevante al tema, como el nivel socio-económico familiar, tipos de apego, autoestima de madres y niños, estilos parentales, entre otras. Asimismo se podría incorporar el informe del padre para, de esta forma, poder establecer si el sexo del progenitor incide en mayor o menor medida que el de la madre y, a su vez, si también padres y madres influyen en niños y niñas de 
modo diferente, ya que en el presente trabajo se observó que las madres influían de modo diferente en algunos dominios del autoconcepto, según el sexo del niño.

Además, podría realizarse un estudio de forma longitudinal con el fin de ver distintas trayectorias en el autoconcepto, y saber cómo cambia la percepción del sí mismo de la niñez a la adolescencia en mujeres y varones. Esto permitiría observar la direccionalidad de la causalidad y distinguir si los cambios entre ambas etapas de la vida se deben a los cambios de edad o al efecto de la cohorte.

En quinto lugar, se sugiere reevaluar los instrumentos, ya que se observó, en el caso del cuestionario de Harter para niños, la confusión o falta de compresión a la hora de su interpretación. Con respecto a esto, Harter planteó dicho formato de respuesta para disminuir la deseabilidad social, pero Wichstrom (1998) halló que esto no sucede y que -por el contrario- dificulta la comprensión de las preguntas. Como ejemplo de lo anteriormente planteado, Rodríguez et al. (2012) y Moreno, Rodríguez y Resett (2012) realizaron una adaptación de este instrumento en niños modificando el formato original de Harter y Pike (1984), y observaron que el mismo conservaba sus propiedades psicométricas.

En último lugar, se sugiere emplear otros métodos de recolección de datos que permitan la obtención de información complementaria, como por ejemplo la realización de entrevistas y/u observaciones en niños. Asimismo, podría incorporarse junto con el informe de los padres, el informe de los docentes con respecto a los niños para comparar el informe de los niños con los de los restantes informantes.

\section{Referencias}

Argyle, M. (1994). The Psychology of Social Class. Nueva York y Londres: Routledge.

Arnett, J. J. (2010). Adolescencia y adultez emergente. México: Pearson.

Atienza F. L., Balaguer I., \& Moreno, Y. (2002). El perfil de autopercepciones para niños: análisis de la validez factorial y la fiabilidad en la versión castellana. Psicothema, 14(3), 659-664.

Baron, A. S., Schmader, T., Cvencek, D., \& Meltzoff, A. N. (2014). The gendered self-concept: How implicit gender stereotypes and attitudes shape selfdefinition. En P. J. Leman \& H. R. Tenenbaum (Eds.), Gender and development (pp. 109-132). East Sussex, Inglaterra: Psychology Press.

Bowlby, J. (1982). Attachment and loss. Vol. 1. Nueva York: Basic Books

Carr, A. (2007). Psicología Positiva. La ciencia de la felicidad. Madrid: Paidós. 
Casullo, M. M. (1990). El autoconcepto. Buenos Aires: Psicoteca.

Collins, W. A., Maccoby, E. E., Steinberg, L., Hetherington, E. M. \& Bornstein, M. H. (2000). Contemporary Research on Parenting: The Case for Nature and Nurture. American Psychologist, 55(2), 218-232.

Davidov, M. \& Grusec, J. E. (2006). Untangling the links of parental responsiveness to distress and warmth to child outcomes. Child Development, 77, 44-58.

Ekman, P. (1972). Universal and cultural differences in facial expressions of emotion. En J. Cole (Ed.), Nebraska Symposyum on Motivation (pp. 207-283). Lincoln: University of Nebraska Press.

Emmons, R. A., \& McCullough, M., E. (2003). Counting Blessings Versus Burdens: An experimental Investigatios of Gratitude and Subjective Well-Being in Daily Life. Journal of Personality and Social Psychology, 84(2), 377-389.

Erikson, E. (1983). Infancia y Sociedad. Buenos Aires: Paidós.

Facio, A., Resett, S., Mistrorigo, C. \& Micocci, F. (2006). Adolescentes Argentinos. Cómo piensan y sienten. Buenos Aires: Lugar Editorial.

Feingold, A. \& Mazzella, R. (1998). Gender differences in body image are increasing. Psychological Science, 9, 190-195.

Fredrickson, B. L. (1998). What good are positive emotions? Review of General Psychology, 2(3), 300-319.

Fredrickson, B. L. (2000). Cultivating positive emotions to optimize health and well-being. Prevention and Treatment, 3, 17-26.

Fredrickson, B. L. (2001). The role of positive emotion in positive psychology: The broaden and build theory of positive emotion. American Psychologist, 56(3), 218-226.

Harter, S. (1985). Manual for the Self-Perception Profile for Children. Denver: University of Denver Press.

Harter, S. (1988). Manual for the Self-Perception Profile for Adolescents. Denver: University of Denver Press.

Harter, S. (1999). The construction of the self. A developmental Perspective. Nueva York - Londres: The Guilford Press. 
Harter, S. (2012). The construction of the self. A developmental Perspective. Segunda Edición. Nueva York - Londres: The Guilford Press.

Harter, S. \& Pike, R. (1984). The Pictorial Scale of Perceived Competence and Social Acceptance for Young Children. Denver: University of Denver Press.

Hattie, J. A. (1992). Self-concept. Hillsdale: Lawrence Erlbaum

Higgins, E. T. (1991). Development of self-regulatory and self-evaluative processes: Costs, benefits, and tradeoffs. En M. R. Gunnar \& L. A. Sroufe (Eds.), Self processes and development. The Minnesota symposia on child psychology (vol. 23, pp. 125-166). Hillsdale: Lawrence Erlbaum Associates.

Higgins, E. (1996). The "self digest": Self-knowledge serving self-regulatory functions. Journal of Personality and Social Psychology, 71(6), 1062-1083.

Jacobs, J., Lanza, S., Osgood, W., Eccles, J. \& Wigfield, A. (2002). Changes in Children's Self-Competence and Values: Gender and Domain Differences across. Child Development, 73(3), 509-527.

Kling, K. C., Hyde, J. S., Showers, C. J. \& Buswell, B. N. (1999). Gender differences in self-esteem: A meta-analysis. Psychological Bulletin, 125, 470-500.

Lazarus, R. S. (2000). Estrés y Emoción. Manejos e implicaciones en nuestra salud. España: Decleé de Brouwer.

Lazarus, R. S. \& Lazarus, B. N. (2000). Pasión y Razón. La comprensión de nuestras emociones. Barcelona: Paidós.

Leaper, C. \& Friedman, C. K. (2007). The socialization of gender. En J. Grusec \& P. Hasting (Eds.), Handbook of socialization (pp. 561-587). Nueva York: Guildford Press.

Lucas, R., Diener, E. \& Larsen, J. (2003). Measuring positive emotions. En S. J. Lopez \& C. R. Snyder (Eds.), Positive psychological assessment: A handbook of models and measures (pp. 201-218). Washington DC: American Psychological Association.

McKay, M. \& Fanning, P. (1991). Autoestima: evaluación y mejora. España: Martínez Roca.

Molina, M. F. \& Raimundi, M. J. (2011). Predictores de la autoestima global en niños de escuela primaria de la Ciudad de Buenos Aires. Diferencias en función del sexo y la edad. Revista Argentina de Ciencias del Comportamiento, 3, 1-7. 
Molina, M. F., Raimundi, M. J., López, C., Cataldi, S. \& Bugallo, L. (2011). Adaptación del Perfil de Autopercepciones para Niños para su uso en la ciudad de Buenos Aires. Revista Iberoamericana de Diagnóstico y Evaluación Psicológica, 32(2), 53- 78.

Moreno, E., Rodríguez, L. \& Resett, S. (2012, noviembre). Diferencias en el autoconcepto y la autoestima en niños de edad escolar. Trabajo presentado en las XIX Jornadas de Investigación de la Facultad de Psicología de la Universidad de Buenos Aires (UBA) y Octavo encuentro de investigadores del Mercosur, Buenos Aires, Argentina.

Nesse, R. \& Ellsworth, P. (2009). Evolution, Emotions, and Emotional Disorders. American Psychologist, 64, 129-139.

Parke, R. \& Gauvain, D. (2009) Child Psychology: A Contemporary View Point. Nueva York: McGraw-Hill.

Regner, E. R. (2009). Emociones positivas como recurso psicológico para el afrontamiento de la amenaza. Tesis de Doctorado en Psicología. Universidad Católica Argentina, Facultad de Psicología y Educación, Buenos Aires, Argentina.

Roberts, K. \& Cunningham, G. (1990). Serenity: Concept analysis and measurement. Educational Gerontology, 16, 577-589.

Richaud de Minzi, M. C. (2006, julio). Children perception of parental empathy in relation with children empathy and social skills. Trabajo presentado en el XVII Congreso de Psicología Aplicada, Atenas, Grecia.

Rodríguez, L., Moreno, J. E. \& Resett, S. (2012, noviembre). El autoconcepto y la autoestima en la niñez escolar. Diferencias según sexo. Trabajo presentado en las Jornadas de Investigación de la UBA, Buenos Aires.

Seligman, M. (2007). La auténtica felicidad. España: Liberdúplex. S.L.U.

Steinberg, L. (2008). Adolescence (8va edición). Nueva York: Mc Graw Hill.

Wichstrom, L. (1998). Self-concept Development during Adolescence. Personality Development in Adolescense. Londres: Routledge. 